\title{
PREVALENCIA DEL BLOQUEO INTERAURICULAR AVANZADO EN PACIENTES REVERTIDOS DE FIBRILACIÓN AURICULAR
}

\author{
INTERATRIAL BLOCK PREVALENCE IN PATIENTS WITH REVERTED ATRIAL \\ FIBRILLATION
}

\section{FLORENCIA FORESSI' ${ }^{1}$ VICTORIA HOYLE², FLORENCIA TRAFICANTE ${ }^{3}$, ROMINA DEGANUTTO ${ }^{4}$, M. BELÉN CIGALINI ${ }^{4}$, J. MARTÍN GALIANO5, CARLOS DUMONT ${ }^{4}$, JORGE GARGUICHEVICH ${ }^{4}$}

\section{RESUMEN}

Introducción. El bloqueo interauricular (BIA) representa un sustrato anatomoeléctrico para el desarrollo de arritmias supraventriculares. Su prevalencia en pacientes con fibrilación auricular (FA) no ha sido claramente estudiada. El objetivo de este trabajo es determinar la prevalencia de BIA en pacientes internados por FA y revertidos a ritmo sinusal.

Métodos. Estudio observacional, analítico, prospectivo, unicéntrico. Se incluyeron pacientes internados por fibrilación auricular (FA) y que hubieran presentado en cualquier momento de su evolución reversión a ritmo sinusal. Post reversión inmediata se realizó un electrocardiograma. A todos los pacientes se les realizó un ecocardiograma transtorácico para evaluar el tamaño de la aurícula izquierda (Al).

Resultados. Se incluyeron 48 pacientes, con una edad promedio de 67,7 años y $56,2 \%$ de sexo masculino. Veintiún pacientes $(43,7 \%)$ presentaron historia previa de FA. El 72,9\% de los pacientes fueron revertidos por cardioversión eléctrica, $12,5 \%$ farmacológica y $14,6 \%$ espontánea. El tamaño de la Al por ecocardiografía fue normal en el 6,2\% de los pacientes, levemente dilatada en el $62,5 \%$, moderada en el $27,1 \%$ y severa en el 4,2\%. La duración promedio de la onda P fue de $128,1 \mathrm{~ms}$. La presencia de componente negativo en las 3 derivaciones de la cara inferior solo se observó en el 4,2\% de los pacientes.

Conclusión. En nuestra población, la incidencia de BIA en pacientes revertidos de FA fue baja.

Palabras clave: bloqueo interauricular; fibrilación auricular; arritmias cardíacas.

\section{ABSTRACT}

Introduction. Interatrial block (IAB) represents ananatomo electric substrate for the development of supraventricular arrhythmias. Its prevalence in patients with atrial fibrillation (AF) has not been clearly studied. The objective of this study is to determine the prevalence of $I A B$ in patients hospitalized for AF and reverted to sinus rhythm.

Methods. Observational, analytical, prospective, single-center study. We included patients admitted for atrial fibrillation (AF) and who presented at any time during their evolution a sinus rhythm reversion. After immediate reversal, an electrocardiogram was performed. All patients underwent a transthoracic echocardiogram to evaluate the size of the left atrium (LA).

Results. 48 patients were included, with an average age of 67.7 years. $56.2 \%$ were males. Twenty-one patients (43.7\%) presented a history of AF. $72.9 \%$ of patients were reversed by electrical cardioversion, $12.5 \%$ pharmacological and $14.6 \%$ spontaneous. The size of the LA by echocardiography was normal in $6.2 \%$ of the patients, slightly dilated in $62.5 \%$, moderate in $27.1 \%$ and severe in $4.2 \%$. The average duration of the $\mathrm{P}$ wave was $128.1 \mathrm{msec}$. The presence of a negative component in the 3 branches of the inferior side was only observed in $4.2 \%$ of the patients.

Conclusions. In our population, the incidence of IAB in patients with AF was low.

Keywords: interatrial block; atrial fibrillation; cardiac arrhythmias.

REVISTA CONAREC 2018;33(145):176-178 | DOI:10.32407/RCON/2018145/0176-0178

\section{INTRODUCCIÓN}

El hallazgo de ondas $P$ ensanchadas y con muescas es muy frecuente en el curso evolutivo de muchas cardiopatías, pudiéndose explicar en base a un crecimiento auricular izquierdo. Sin embargo, una onda $P \geq 120$ ms no implica exclusivamente el concepto de agrandamiento auricular izquierdo ya que puede tratarse de un bloqueo interauricular (BIA); de hecho, ambas entidades suelen coexistir y los criterios electrocardiográficos superponerse ${ }^{1-3}$. Tanto en nuestra experien-

1. Jefe de residentes de Cardiología.

2. Residente de Cardiología

3. Instructor de residentes de Cardiología.

4. Médico de planta de Cardiología.

5. Ex residente de Cardiología.

Hospital Privado de Rosario. Rosario, Santa Fe, Rep. Argentina

$\triangle$ Correspondencia: Dra. Florencia Foressi. Dorrego 59 Bis 7 mo B. CP 2000. Rosario Santa Fe. Argentina.floforessi@gmail.com.

Los autores declaran no poseer conflictos de intereses.

Recibido: 11/10/2017 | Aceptado: 27/08/2018 cia como en la de otros autores, la presencia de "muescas" en la onda $P$ es un dato a considerar, ya que suele acompañar frecuentemente a los BIA 4,5 .

El BIA representa un sustrato anatomoeléctrico para el desarrollo de arritmias supraventriculares, en particular fibrilación auricular (FA), como bien lo describió a fines de la década de los setenta el Dr. Bayés de Luna, hoy conocido como síndrome de Bayés ${ }^{6-9}$.

Los BIA pueden clasificarse en parcial (Figura 1), donde el retraso existiría en la zona del haz de Bachmann (evidenciado por una onda $P \geq 120 \mathrm{~ms}$, con o sin muescas, sin componente negativo final en las derivaciones inferiores), y en avanzado (Figura 2), donde la despolarización de la aurícula izquierda (Al) se produce por la estimulación a partir de la aurícula derecha (por el seno coronario y en menor parte por la fossa ovalis) con una dirección retrógrada caudocraneal, generando una onda $\mathrm{P} \geq 120$ mseg con la presencia de un componente negativo final en las 3 derivaciones inferiores.

Además de los trabajos de Bayés de Luna, diferentes investigadores han demostrado que el BIA avanzado representa un marcador de riesgo de FA o de recurrencia de la misma ${ }^{10-14}$. Su prevalencia en pacientes con FA no ha sido claramente determinada. La mayoría de los estudios incluyen en forma conjunta la presencia de BIA parcial y avanzado. 


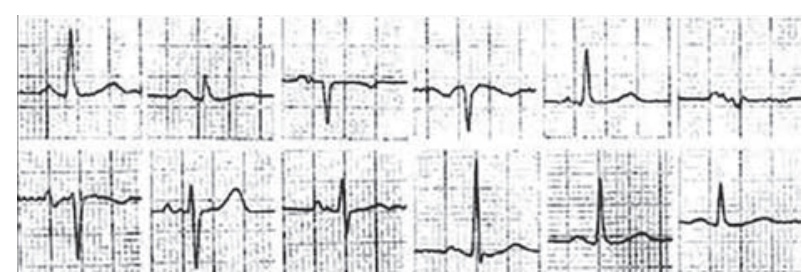

Figura 1. Bloqueo interauricular parcial. BIA: bloqueo interauricular. FA: fibrilación auricular. Modificado de: Cigalini MB, Deganutto R, Galiano JM, Foressi F, Sanziani L, Cardona M, et al. Evaluación de los marcadores electrocardiográicos de riesgo de fibrilación auricular en pacientes sin cardiopatía estructural significativa. Rev Fed Arg Cardiol 2015;44(4):29-33.

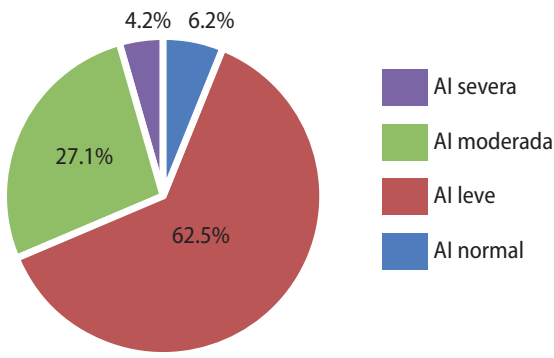

Figura 3. Distribución acorde a tamaño de la aurícula izquierda. Al: aurícula izquierda.

El objetivo de este trabajo es determinar la prevalencia de BIA avanzado en pacientes internados por FA y revertidos a ritmo sinusal, ampliando los registros electrocardiográficos de forma digital, para analizar mejor la morfología de la onda P.

\section{MATERIALES Y MÉTODOS}

Se realizó un estudio observacional, analítico, prospectivo, unicéntrico, desde julio de 2016 hasta febrero de 2017. Se incluyeron pacientes de cualquier edad, sexo y patología, internados por FA en nuestra Unidad Coronaria y que hayan presentado en cualquier momento de su evolución reversión a ritmo sinusal. Inmediatamente posreversión se realizó un electrocardiograma de 12 derivaciones con voltaje habitual, que luego fue ampliado de forma digital. A todos los pacientes se les realizó un ecocardiograma transtorácico para evaluar el tamaño de la Al.

Las variables discretas se expresaron como porcentajes y las continuas, según su distribución, como media y desvío estándar (DE) o mediana y rango intercuartilo.

\section{CONSIDERACIONES ÉTICAS}

El Comité de Docencia e Investigación institucional aprobó el presente trabajo y todos los pacientes han dado su consentimiento por escrito. El trabajo respeta las propuestas de la Declaración de Helsinki.

\section{RESULTADOS}

Se incluyeron 48 pacientes, con una edad promedio de 67,7 años (3489) el 56,2\% de sexo masculino. Veintiún pacientes $(43,7 \%)$ presentaron historia previa de FA.

La patología predominante fue la hipertensión arterial (58,3\%) (Tabla 1). El tamaño de la Al por ecocardiografía fue normal en 3 pacientes $(6,2 \%)$, dilatada de manera leve en 30 (62,5\%), moderada en 13

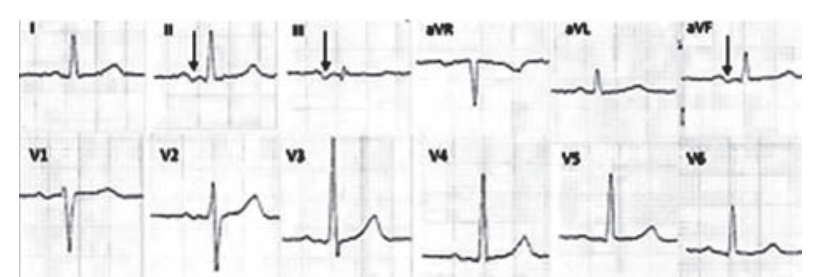

Figura 2. Bloqueo interauricular avanzado. BIA: bloqueo interauricular. FA: fibrilación auricular. Modificado de: Cigalini MB, Deganutto R, Galiano JM, Foressi F, Sanziani L, Cardona M, et al. Evaluación de los marcadores electrocardiográficos de riesgo de fibrilación auricular en pacientes sin cardiopatía estructural significativa. Rev Fed Arg Cardiol 2015;44(4):29-33.

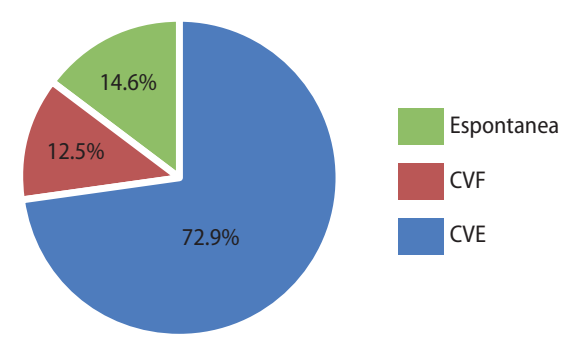

Figura 4. Distribución acorde a tipo de reversión de fibrilación auricular. FA: fibrilación auricular. CVE: cardioversión eléctrica. CVF: cardioversión farmacológica

Tabla 1. Características basales $(n=48)$.

\begin{tabular}{|c|c|}
\hline Edad media (años) & 67,7 \\
\hline Sexo masculino - n (\%) & $27(56,2 \%)$ \\
\hline Historia previa de FA - n (\%) & $21(43,7 \%)$ \\
\hline Al normal - n (\%) & $3(6,2 \%)$ \\
\hline Agrandamiento Al leve - n (\%) & $30(62.5 \%)$ \\
\hline Agrandamiento Al moderado - n (\%) & $13(27,1 \%)$ \\
\hline Agrandamiento Al severo - $\mathrm{n}(\%)$ & $2(4,2 \%)$ \\
\hline HTA - n (\%) & $28(58,3 \%)$ \\
\hline Cardiopatía isquémica - n (\%) & $9(18,7 \%)$ \\
\hline Valvulopatía - n (\%) & $7(14,5 \%)$ \\
\hline AlT - n (\%) & $2(4,2 \%)$ \\
\hline Enfermedad vascular periférica - n (\%) & $3(6,2 \%)$ \\
\hline Sin cardiopatía estructural - n (\%) & $12(25 \%)$ \\
\hline
\end{tabular}

FA: fibrilación auricular. Al: aurícula izquierda. HTA: hipertensión arterial. AlT: accidente isquémico transitorio.

$(27,1 \%)$ y severa en $2(4,2 \%)$ (Figura 3). Treinta y cinco pacientes $(72,9 \%)$ fueron revertidos por cardioversión eléctrica, 6 (12,5\%) farmacológica y 7 (14,6\%) lo hicieron de forma espontánea (Figura 4). La duración promedio de la onda P fue de 128,1 ms (120-200), 39 de las cuales $(81,25 \%)$ presentaron muescas. La presencia de componente negativo en las 3 derivaciones de la cara inferior solo se observó en 2 pacientes (4,2\%), 1 de ellos con historia previa de FA. En 6 pacientes (12,5\%) se registró en dos derivaciones (predominando D III y aVF), 2 de ellos con antecedentes de FA. En 10 pacientes (20,8\%) se visualizó en una sola derivación (D III en el 90\% de los casos).

\section{DISCUSIÓN}

En nuestra población de pacientes recientemente revertidos de FA, la prevalencia de BIA avanzado fue baja (4,2\%).

Hemos encontrado un solo trabajo similar efectuado en 61 pacientes re- 
vertidos farmacológicamente de FA. En él la prevalencia del BIA avanzado fue del $18 \%$ con una recurrencia de FA en estos pacientes del $90 \%$ al año ${ }^{11}$. EI BIA existe como entidad anatomoeléctrica y debe ser considerado como un verdadero bloqueo, con diferentes grados, al igual que los otros bloqueos del sistema de conducción. Se encuentra fuertemente asociado con arritmias supraventriculares y es probablemente un predictor de accidente cerebrovascular cardioembólico ${ }^{15-18}$. Un simple electrocardiograma permite la identificación inmediata de la patente de BIA avanzado.

La limitante principal de nuestro estudio ha sido el bajo de número de pacientes. Es probable que estudios de mayores dimensiones, que

\section{BIBLIOGRAFÍA}

1. Tsao CW, Josephson ME, Hauser TH, O'Halloran TD, Agarwal A, Manning WJ, et al. Accuracy of electrocardiographic criteria for atrial enlargement: validation with cardiovascular magnetic resonance. J Cardiovasc Magn Reson 2008;10(1):7.

2. Spodick DH, Ariyarajah V. Interatrial block: the pandemic remains poorly perceived. Pacing Clin Electrophisiol 2009:32(5):667-72.

3. Galiano J, Deganutto R, Cigalini M, Foressi F, Hoyle V, Dumont D, et al. Precisión de los criterios electrocardiográficos para el diagnóstico de agrandamiento auricular izquierdo. ¿Agrandamiento auricular izquierdo o trastorno de conducción auricular? (Octubre de 2015). Poster presentado en XXXIV Congreso Nacional de Cardiología.

4. Ariyarajah V, Apiyasawat $S$, Puri P, Spodick DH. Specific electrocardiographic markers of P-wave morphology in interatrial block. J Electrocardiol 2006;39(4):380-4.

5. Cigalini MB, Deganutto $R$, Galiano JM, Foressi F, Sanziani L, Cardona $M$, et al. Evaluación de los marcadores electrocardiográficos de riesgo de fibrilación auricular en pacientes sin cardiopatía estructural significativa. Rev Fed Arg Cardiol 2015:44(4):29-33.

6. Bayés de Luna A. Bloqueo a nivel auricular. Rev Esp Cardiol 1979;32(1):5-10

7. Bayés de Luna A, Fort de Ribot R, Trilla E, Julia J, García J, Sadurni J, et al. Electrocardiographic and vector cardiographic study of interatrial conduction disturbances with left atrial retrograde activation. J Electrocardiol 1985; 18(1):1-13.

8. Bayés de Luna A, Platonov P, Cosio FG, Cygankiewicz I, Pastore C, Baranowski R, et al. Interatrial blocks. A separate entity from left atrial enlargement: a consensus report. J Electrocardiol 2012;45(5):445-51.

9. Conde D, Baranchuk A. Bloqueo interauricular como sustrato anatómico-eléctrico de arritmias supraventriculares: sindrome de Bayés. Arch Cardiol Mex 2014;84(1):32-40 incluyan además pacientes con mayor patología, puedan demostrar otra prevalencia.

\section{CONCLUSIÓN}

En nuestra población, la incidencia de BIA avanzado en pacientes revertidos de FA fue baja. A pesar de esto, su pronto reconocimiento ayudaría a un monitoreo cardíaco más intensivo en busca de FA y a la iniciación más temprana de anticoagulación y/o tratamiento antiarrítmico, ayudándonos de esa manera a prevenir accidentes cerebrovasculares y sus devastadoras consecuencias.

10. Caldwell JC, Koppikar S, Barake W, Redfearn DP, Michael K, Simpson C, et al. Advanced interatrial block is associated with atrial fibrillation recurrence after successful pulmonary vein isolation for paroxysmal atrial fibrillation. J Electrocardiol 2013;46(1):e1.

11. Enríquez A, Conde D, Hopman W, Mondragon I, Chiale PA, De Luna AB, et al. Advanced interatrial block is associated with recurrence of atrial fibrillation post pharmacological cardioversion. Cardiovasc Ther 2014;32(2):52-6.

12. Enríquez A Conde D, Femenía F De Luna AB, Ribeiro A, Muratore C et al Relation of interatrial block to new-onset atrial fibrillation in patients with Chagas cardiomyopathy and implantable cardioverter defibrillators. Am J Cardiol 2014:113(10):1740-3

13. Enríquez A, Sarrias A, Villuendas R, Ali FS, Conde D, Hopman WM, et al. New-onset atrial fibrillation after cavotricuspid isthmus ablation: identification of advanced interatrial block is key. Europace 2015;17(8):1289-93.

14. Tekkesin Al, Çinier G, Cakilli Y, Hayıroğlu MI, Alper AT. Interatrial block predicts atri al high rate episodes detected by cardiac implantable electronic devices. J Electrocardiol 2017:50(2):234-7.

15. Ariyarajah V, Apiyasawat S, Najjar H, Mercado K, Puri P, Spodick DH. Frequency of interatrial block in patients with sinus rhythm hospitalized for stroke and comparison to those without interatrial block. Am J Cardiol 2007:99(1):49-52.

16. Ariyarajah V, Puri P, Apiyasawat S, Spodick DH. Interatrial block: A novel risk factor for embolic stroke? Ann Noninvasive Electrocardiol 2007;12(1):15-20.

17. Martinez-Selles M, Baranchuk A, Elosua R, Bayes de Luna A, O'neal WT, Kamel H, et al. Advanced interatrial block and ischemic stroke: The Atherosclerosis Risk In Communities Study. Neurology 2016;87(23):2499.

18. Martinez-Selles M, Escobar LA, Baranchuk A. Interatrial block and the risk of ischemic stroke. J Atheroscler Thromb 2017;24(2):185-6. 